\title{
TESS Discovery of a Transiting Super-Earth in the pi Mensae System
}

Huang, Chelsea X.; Burt, Jennifer; Vanderburg, Andrew; Günther, Maximilian N.; Shporer, Avi; Dittmann, Jason A.; Winn, Joshua N.; Wittenmyer, Rob; Sha, Lizhou; Kane, Stephen R.

Total number of authors:

50

Published in:

Astrophysical Journal Letters

Link to article, DOI:

10.3847/2041-8213/aaef91

Publication date:

2018

Document Version

Publisher's PDF, also known as Version of record

Link back to DTU Orbit

Citation $(A P A)$ :

Huang, C. X., Burt, J., Vanderburg, A., Günther, M. N., Shporer, A., Dittmann, J. A., Winn, J. N., Wittenmyer, R., Sha, L., Kane, S. R., Ricker, G. R., Vanderspek, R. K., Latham, D. W., Seager, S., Jenkins, J. M., Caldwell, D. A., Collins, K. A., Guerrero, N., Smith, J. C., ... Buchhave, L. A. (2018). TESS Discovery of a Transiting SuperEarth in the pi Mensae System. Astrophysical Journal Letters, 868(2), [L39]. https://doi.org/10.3847/2041$8213 /$ aaef91

\section{General rights}

Copyright and moral rights for the publications made accessible in the public portal are retained by the authors and/or other copyright owners and it is a condition of accessing publications that users recognise and abide by the legal requirements associated with these rights.

- Users may download and print one copy of any publication from the public portal for the purpose of private study or research.

- You may not further distribute the material or use it for any profit-making activity or commercial gain

- You may freely distribute the URL identifying the publication in the public portal 


\title{
TESS Discovery of a Transiting Super-Earth in the pi Mensae System
}

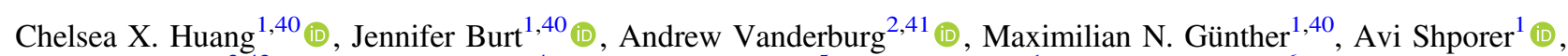
Jason A. Dittmann ${ }^{3,42}$ (1) , Joshua N. Winn ${ }^{4}$ (1), Rob Wittenmyer ${ }^{5}$ (10), Lizhou Sha ${ }^{1}$, Stephen R. Kane ${ }^{6}(\mathbb{1})$, George R. Ricker ${ }^{1}$, Roland K. Vanderspek ${ }^{1}$ (i), David W. Latham ${ }^{7}$ (1) , Sara Seager ${ }^{1,3}$, Jon M. Jenkins ${ }^{8}$ (1), Douglas A. Caldwell ${ }^{8,9}$, Karen A. Collins ${ }^{7}$ (1), Natalia Guerrero ${ }^{1}$, Jeffrey C. Smith ${ }^{9}$, Samuel N. Quinn ${ }^{8}$ (1), Stéphane Udry ${ }^{10}$, Francesco Pepe ${ }^{10}$, François Bouchy ${ }^{10}$, Damien Ségransan $^{10}$, Christophe Lovis $^{10}$, David Ehrenreich ${ }^{10}$ (10), Maxime Marmier ${ }^{10}$, Michel Mayor ${ }^{10}$, Bill Wohler ${ }^{8,9}$, Kari Haworth ${ }^{1}$, Edward H. Morgan ${ }^{1}(\mathbb{1})$, Michael Fausnaugh ${ }^{1}$, David R. Ciardi ${ }^{11}$, Jessie Christiansen ${ }^{11}$ (1), David Charbonneau ${ }^{7}$ (1),

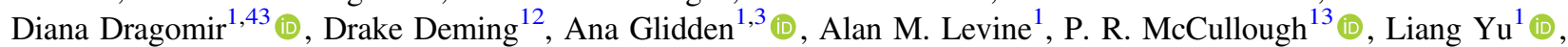

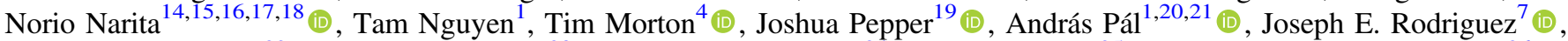
Keivan G. Stassun ${ }^{22}$ (1) , Guillermo Torres ${ }^{23}$ (1), Alessandro Sozzetti ${ }^{24}$ (D), John P. Doty ${ }^{25}$, Jørgen Christensen-Dalsgaard ${ }^{26}$, Gregory Laughlin ${ }^{27}$ (1), Mark Clampin ${ }^{28}$, Jacob L. Bean ${ }^{29}$, Lars A. Buchhave ${ }^{30}$, G. Á. Bakos ${ }^{31,32,44}$ (1) , Bun'ei Sato $^{33}$, Shigeru Ida ${ }^{34}$, Lisa Kaltenegger $^{35}$, Enric Palle ${ }^{36}$, Dimitar Sasselov ${ }^{23}$ (D) R. P. Butler ${ }^{37}$, Jack Lissauer ${ }^{38}$, Jian Ge ${ }^{39}$, and S. A. Rinehart ${ }^{28}$

${ }^{1}$ Department of Physics, and Kavli Institute for Astrophysics and Space Research, Massachusetts Institute of Technology, Cambridge, MA 02139, USA

${ }^{2}$ Department of Astronomy, The University of Texas at Austin, Austin, TX 78712, USA

${ }^{3}$ Earth and Planetary Sciences, MIT, 77 Massachusetts Avenue, Cambridge, MA 02139, USA

${ }^{4}$ Department of Astrophysical Sciences, Princeton University, 4 Ivy Lane, Princeton, NJ 08544, USA

${ }^{5}$ University of Southern Queensland, West Street, Darling Heights QLD 4350, Australia

${ }^{6}$ Department of Earth Sciences, University of California, Riverside, CA 92521, USA

${ }^{7}$ Harvard-Smithsonian Center for Astrophysics, Harvard University, Cambridge, MA 02138, USA

${ }^{8}$ NASA Ames Research Center, Moffett Field, CA, 94035, USA

${ }^{9}$ SETI Institute, Mountain View, CA 94043, USA

${ }^{10}$ Observatoire de l'Université de Genève, 51 chemin des Mail-lettes, 1290 Versoix, Switzerland

${ }^{11}$ NASA Exoplanet Science Institute, Caltech/IPAC-NExScI, 1200 East California Boulevard, Pasadena, CA 91125, USA

${ }_{12}^{2}$ Department of Astronomy, University of Maryland at College Park, College Park MD, 20742, USA

${ }^{13}$ Department of Physics and Astronomy, Johns Hopkins University, 3400 North Charles Street, Baltimore, MD 21218, USA

${ }^{14}$ Department of Astronomy, The University of Tokyo, 7-3-1 Hongo, Bunkyo-ku, Tokyo 113-0033, Japan

${ }^{15}$ Astrobiology Center, National Astronomical Observatory of Japan, NINS, 2-21-1 Osawa, Mitaka, Tokyo 181-8588, Japan ${ }^{16}$ JST, PRESTO, 7-3-1 Hongo, Bunkyo-ku, Tokyo 113-0033, Japan

${ }^{17}$ Instituto de Astrofísica de Canarias (IAC), E-38205 La Laguna, Tenerife, Spain

18 National Astronomical Observatory of Japan, NINS, 2-21-1 Osawa, Mitaka, Tokyo 181-8588, Japan

19 Department of Physics, Lehigh University, 16 Memorial Drive East, Bethlehem, PA 18015, USA

${ }^{20}$ Department of Astronomy, Loránd Eötvös University, Pázmány P. stny. 1/A, Budapest H-1117, Hungary

${ }^{21}$ Konkoly Observatory, Research Centre for Astronomy and Earth Sciences, Hungarian Academy of Sciences,

Konkoly Thege Miklós út 15-17, H-1121 Budapest, Hungary
22 Vanderbilt University, Department of Physics \& Astronomy, 6301 Stevenson Center Ln., Nashville, TN 37235, USA

${ }^{23}$ Harvard-Smithsonian Center for Astrophysics, 60 Garden Street, Cambridge, MA 02138, USA

${ }^{24}$ INAF-Osservatorio Astrofisico di Torino, Via Osservatorio 20, 10025 Pino Torinese, Italy

${ }^{25}$ Noqsi Aerospace Ltd., 2822 South Nova Road, Pine, CO 80470, USA

${ }^{26}$ Stellar Astrophysics Centre, Department of Physics and Astronomy, Aarhus University, Ny Munkegade 120, DK-8000 Aarhus C, Denmark ${ }_{27}$ Department of Astronomy, Yale University, New Haven, CT 06517, USA

${ }^{28}$ NASA Goddard Space Flight Center, 8800 Greenbelt Road, Greenbelt, MD 20771, USA

${ }^{29}$ Department of Astronomy \& Astrophysics, University of Chicago, 5640 South Ellis Avenue, Chicago, IL 60637, USA

${ }^{30}$ DTU Space, National Space Institute, Technical University of Denmark, Elektrovej 328, DK-2800 Kgs. Lyngby, Denmark ${ }^{31}$ Department of Astrophysical Sciences, Princeton University, NJ 08544, USA

${ }^{32}$ MTA Distinguished Guest Fellow, Konkoly Observatory, Hungary

${ }^{33}$ Department of Earth and Planetary Sciences, Tokyo Institute of Technology, 2-12-1 Ookayama, Meguro-ku, Tokyo 152-8551, Japan

${ }_{35}$ Earth-Life Science Institute, Tokyo Institute of Technology, Ookayama, Meguro-ku, Tokyo 152-8550, Japan

${ }^{35}$ Carl Sagan Institute at Cornell University, 302 Space Science Building, Ithaca, NY 14853, USA

${ }^{36}$ Instituto de Astrofisica de Canarias, Via Lactea sn, 38200, La Laguna, Tenerife, Spain

${ }^{37}$ Carnegie Institution for Science, Department of Terrestrial Magnetism, 5241 Broad Branch Road NW, Washington, DC 20015, USA

${ }^{38}$ Space Science \& Astrobiology Division, NASA Ames Research Center, Moffett Field, CA 94035, USA

${ }^{39}$ Astronomy Department, University of Florida, 211 Bryant Space Science Center, P.O. Box 112055, Gainesville, FL 32611-2055, USA

Received 2018 September 16; revised 2018 November 7; accepted 2018 November 7; published 2018 November 30

\begin{abstract}
We report the detection of a transiting planet around $\pi$ Men (HD 39091), using data from the Transiting Exoplanet Survey Satellite (TESS). The solar-type host star is unusually bright $(V=5.7)$ and was already known to host a Jovian planet on a highly eccentric, $5.7 \mathrm{yr}$ orbit. The newly discovered planet has a size of $2.04 \pm 0.05 R_{\oplus}$ and an
\end{abstract}

\footnotetext{
40 Juan Carlos Torres Fellow.

41 NASA Sagan Fellow.

4251 Pegasi b Postdoctoral Fellow.

43 NASA Hubble Fellow.

44 Packard Fellow.
} 
orbital period of 6.27 days. Radial-velocity data from the High-Accuracy Radial-velocity Planet Searcher and Anglo-Australian Telescope/University College London Echelle Spectrograph archives also displays a 6.27 day periodicity, confirming the existence of the planet and leading to a mass determination of $4.82 \pm 0.85 M_{\oplus}$. The star's proximity and brightness will facilitate further investigations, such as atmospheric spectroscopy, asteroseismology, the Rossiter-McLaughlin effect, astrometry, and direct imaging.

Key words: planetary systems - planets and satellites: detection - stars: individual (HD 39091, TIC 261136679)

\section{Introduction}

The mission of the Transiting Exoplanet Survey Satellite (TESS; Ricker et al. 2015) is to search for transiting planets as small as Earth around the nearest and brightest stars. Four $10 \mathrm{~cm}$ optical telescopes are used to repeatedly image wide fields and monitor the brightness of suitable stars. The data are then searched for periodic dips that could be caused by transiting planets. The spacecraft was launched on 2018 April 18 and began the sky survey on July 25 . Here, we report on the discovery of a small transiting planet around a bright star $\pi$ Men.

$\pi$ Men (also known as HD 39091) is a naked-eye G0V star at a distance of $18.27 \pm 0.02 \mathrm{pc}$ (Gaia Collaboration et al. 2018) with a mass of $1.1 M_{\odot}$ and a radius of $1.1 R_{\odot}$. Doppler monitoring by Jones et al. (2002) and Wittenmyer et al. (2012) revealed a planet ( $\pi$ Men b) with a mass about 10 times that of Jupiter, an orbital period of $5.7 \mathrm{yr}$, and an orbital eccentricity of 0.6 . With a visual apparent magnitude of 5.67 , the star is a prime target for the TESS survey. It is one of several hundred thousand pre-selected stars for which data will be available with 2 minute time sampling, as opposed to the 30 minute sampling of the full image data set.

This Letter is organized as follows. Section 2 presents the TESS photometric data that led to the detection of the new planet $\pi$ Men c, as well as the archival radial velocity data that confirm the planet's existence. Section 3 describes our methods for determining the system parameters, including the mass and radius of the star and planet. Section 4 discusses some possible follow-up observations that will be facilitated by the star's brightness and proximity to Earth.

\section{Observations and Data Reduction}

\subsection{TESS Photometry}

The TESS survey divides the sky into 26 partially overlapping sectors, each of which is observed for approximately one month during the two-year primary mission. $\pi$ Men is located near the southern ecliptic pole in a region where six sectors overlap, implying that it is scheduled to be observed for a total of 6 months. This Letter is based on data from Sector 1 (2018 July 25-August 22), during which $\pi$ Men was observed with charge-coupled device (CCD) 2 of Camera 4.

The data were processed with two independently written codes: the MIT Quick Look Pipeline (partially based on fitsh; Pál 2009), which analyzes the full images that are obtained with 30 minute time sampling; and the Science Processing Operations Center pipeline, a descendant of the Kepler mission pipeline based at the NASA Ames Research Center (Jenkins et al. 2010), which analyzes the 2 minute data that are obtained for pre-selected target stars. For $\pi$ Men, both pipelines detected a signal with a period of 6.27 days, an amplitude of about $300 \mathrm{ppm}$, a duration of $3 \mathrm{hr}$, and a flat-bottomed shape consistent with the light curve of a planetary transit.

Previous surveys taught us that transit-like signals sometimes turn out to be eclipsing binaries that are either grazing, or blended with a bright star, causing the amplitude of the signal to be deceptively small and resemble that of a planet (e.g., Cameron 2012). In this case, the signal survived all of the usual tests for such "false positives." There is no discernible secondary eclipse, no detectable alternation in the depth of the transits, and no detectable motion of the stellar image on the detector during the fading events. ${ }^{45}$

After identifying the transits, we tried improving on the light curve by experimenting with different choices for the photometric aperture, including circles as well as irregular pixel boundaries that enclose the blooming stellar image. Best results were obtained for the aperture shown in Figure 1. Also shown are images of the field from optical sky surveys conducted 30-40 yr ago, long enough for the star to have moved about an arcminute relative to the background stars. This allows us a clear view along the line of sight to the current position of $\pi$ Men, which is reassuringly blank: another indication that the transit signal is genuine and not an unresolved eclipsing binary. The other stars within the photometric aperture are too faint to cause the $300 \mathrm{ppm}$ fading events.

The top panel of Figure 2 shows the result of simple aperture photometry. Most of the observed variation is instrumental. There may also be a contribution from stellar variability, which is expected to occur on the 18 day timescale of the rotation period (Zurlo et al. 2018). To remove these variations and permit a sensitive search for transits, we fitted a basis spline with knots spaced by 0.3 days, after excluding both $3 \sigma$ outliers and the data obtained during and immediately surrounding transits. We then divided the light curve by the best-fitting spline.

The middle panel of Figure 2 shows the result. The scatter is $142 \mathrm{ppm}$ per 2 minute sample, and $30 \mathrm{ppm}$ when averaged into $6 \mathrm{hr}$ bins, comparable to the highest-quality Kepler light curves. The gap in the middle of the time series occurred when observations were halted for data downlink. The other gap occurred during a period when the spacecraft pointing jitter was higher than normal. We also excluded the data from the 30-60 minute intervals surrounding "momentum dumps," when thrusters are fired to reorient the spacecraft and allow the reaction wheels to spin down. The times of the momentum dumps are marked in Figure 2. There were 10 such events during Sector 1 observations, occurring every $21 / 2$ days.

\subsection{Radial-velocity Data}

$\pi$ Men has been monitored for $20 \mathrm{yr}$ as part of the AngloAustralian Planet Search, which uses the $3.9 \mathrm{~m}$ AngloAustralian Telescope (AAT) and the University College London Echelle Spectrograph (UCLES; Diego et al. 1990). The long-period giant planet $\pi$ Men $\mathrm{b}$ was discovered in this survey (Jones et al. 2002; Butler et al. 2006). A total of 77

\footnotetext{
$\overline{45}$ The last test in the list, the centroid test, was complicated by the fact that the star is bright enough to cause blooming in the TESS CCD images. The associated systematic effects were removed using the method of Günther et al. (2017).
} 

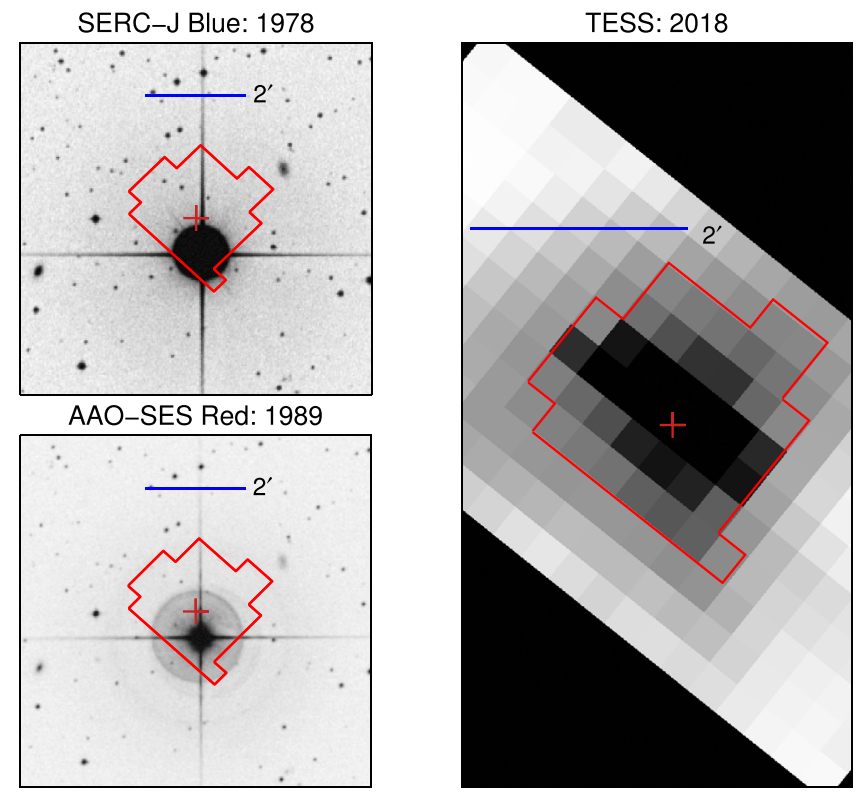

Figure 1. Images of the field surrounding $\pi$ Men. Top-left panel: from the Science and Engineering Research Council $\mathbf{J}$ survey, obtained with a bluesensitive photographic emulsion in 1978. The red cross is the current position of $\pi$ Men. Red lines mark the boundary of the TESS photometric aperture. Bottom-left panel: from the AAO Second Epoch Survey, obtained with a redsensitive photographic emulsion in 1989. Right panel: summed TESS image. North is up and East is to the left in all of the images.

radial velocities are available, obtained between 1998 and 2015 , with a mean internal uncertainty of $2.13 \mathrm{~m} \mathrm{~s}^{-1}$.

The star was also monitored with the High-Accuracy Radialvelocity Planet Searcher HARPS (Mayor et al. 2003) on the ESO $3.6 \mathrm{~m}$ telescope at La Silla Observatory in Chile. A hardware upgrade in 2015 June led to an offset in the velocity scale (Lo Curto et al. 2015). For this reason, our model allows for different constants to be added to the pre-upgrade and postupgrade data. A total of 145 radial velocities are available, obtained between 2003 December and 2016 March with irregular sampling. The mean internal uncertainty of the 128 pre-upgrade velocities is $0.78 \mathrm{~m} \mathrm{~s}^{-1}$, while that of the 17 postupgrade velocities is $0.38 \mathrm{~m} \mathrm{~s}^{-1}$.

The top panel of Figure 3 shows the radial-velocity data. It is easy to see the $400 \mathrm{~m} \mathrm{~s}^{-1}$ variations from the giant planet. To search for evidence of the new planet, we subtracted the bestfitting single-planet model from the data and computed the Lomb-Scargle periodogram of the more precise HARPS data, shown in the middle panel. The highest peak is far above the $0.1 \%$ false alarm threshold and is located at the transit period of 6.27 days. The next highest peaks, bracketing a period of 1 day, are aliases of this signal. The phase of the 6.27 day signal is also consistent with the measured transit times.

We consider this to be a decisive confirmation of the existence of $\pi$ Men c. Still, as another precaution against false positives, we checked the HARPS spectra for any indication of a second star, or spectral-line distortions associated with the 6.27 day signal. We re-analyzed the HARPS cross-correlation functions with the BLENDFITTER routine (Günther et al. 2018) and found no sign of any correlated bisector variations.

\section{Determination of System Parameters}

We performed a joint analysis of the two-planet system using the TESS transit light curve and the 222 radial velocities from
Table 1

System Parameters for $\pi$ Men

\begin{tabular}{|c|c|c|}
\hline Stellar Parameters & Value & Source \\
\hline \multicolumn{3}{|l|}{ Catalog Information } \\
\hline R.A. (h:m:s) & 05:37:09.89 & Gaia Data Release 2 (DR2) \\
\hline Decl. (d:m:s) & $-80: 28: 08.8$ & Gaia DR2 \\
\hline Epoch & 2015.5 & Gaia DR2 \\
\hline Parallax (mas) & $54.705 \pm 0.067$ & Gaia DR2 \\
\hline $\begin{array}{l}\mu_{\mathrm{R} . \mathrm{A} .}(\mathrm{mas} \\
\mathrm{yr}^{-1} \text { ) }\end{array}$ & $311.19 \pm 0.13$ & Gaia DR2 \\
\hline $\begin{array}{l}\mu_{\text {Decl. }}(\text { mas } \\
\left.\mathrm{yr}^{-1}\right)\end{array}$ & $1048.85 \pm 0.14$ & Gaia DR2 \\
\hline Gaia DR2 ID & 4623036865373793408 & \\
\hline HD ID & HD 39091 & \\
\hline TIC ID & 261136679 & Stassun et al. (2018) \\
\hline TOI ID & 144.01 & \\
\hline \multicolumn{3}{|c|}{ Spectroscopic Properties } \\
\hline$T_{\text {eff }}(\mathrm{K})$ & $6037 \pm 45$ & Ghezzi et al. (2010) \\
\hline $\log g(\mathrm{cgs})$ & $4.42 \pm 0.03$ & Ghezzi et al. (2010) \\
\hline$[\mathrm{Fe} / \mathrm{H}](\mathrm{dex})$ & $0.08 \pm 0.03$ & Ghezzi et al. (2010) \\
\hline$v \sin i\left(\mathrm{~km} \mathrm{~s}^{-1}\right)$ & $3.14 \pm 0.50$ & Valenti \& Fischer (2005) \\
\hline \multicolumn{3}{|c|}{ Photometric Properties } \\
\hline$B$ (mag) & 6.25 & \\
\hline$V(\mathrm{mag})$ & 5.67 & \\
\hline TESS (mag) & 5.1 & TIC V7 \\
\hline Gaia (mag) & 5.491 & Gaia DR2 \\
\hline Gaia $_{r}(\mathrm{mag})$ & 5.064 & Gaia DR2 \\
\hline Gaia $_{b}(\mathrm{mag})$ & 5.838 & Gaia DR2 \\
\hline$J$ (mag) & $4.87 \pm 0.27$ & $\begin{array}{c}\text { Two Micron All-Sky } \\
\text { Survey (2MASS) }\end{array}$ \\
\hline$H$ (mag) & $4.42 \pm 0.23$ & 2MASS \\
\hline$K_{s}(\mathrm{mag})$ & $4.241 \pm 0.027$ & 2MASS \\
\hline \multicolumn{3}{|l|}{ Derived Properties } \\
\hline$M_{\star}\left(M_{\odot}\right)$ & $1.094 \pm 0.039$ & This work \\
\hline$R_{\star}\left(R_{\odot}\right)$ & $1.10 \pm 0.023^{\mathrm{a}}$ & This work \\
\hline$L_{\star}\left(L_{\odot}\right)$ & $1.444 \pm 0.02$ & This work \\
\hline Age (Gyr) & $2.98_{-1.3}^{+1.4}$ & This work \\
\hline Distance (pc) & $18.27 \pm 0.02$ & Gaia DR2 \\
\hline$\rho_{\star}\left(\mathrm{g} \mathrm{cm}^{-3}\right)$ & $1.148 \pm 0.065$ & This work \\
\hline
\end{tabular}

Note.

${ }^{\mathrm{a}}$ This is consistent with the radius of star derived from spectra energy analysis by Stassun et al. (2018).

the AAT and HARPS surveys. The orbit of planet $\mathrm{c}$ was assumed to be circular in the fit. ${ }^{46}$ As noted previously, we assigned a different additive constant to each of the three radial-velocity data sets. We also required the systemic velocity to be zero and allowed for three independent values of the "jitter," a term that is added in quadrature to the internally estimated measurement uncertainty to account for systematic effects.

We assumed the star to follow a quadratic limb-darkening law and used the formulas of Mandel \& Agol (2002) as implemented by Kreidberg (2015). We fixed the limbdarkening coefficients at $u_{1}=0.28$ and $u_{2}=0.27$, based on the tabulation of Claret (2017). The photometric model was computed with 0.4 minutes sampling and then averaged to 2 minutes before comparing with the data.

\footnotetext{
46 We also tried allowing planet $\mathrm{c}$ to have an eccentric orbit, which resulted in an upper limit of $e_{c}<0.3(1 \sigma)$. All of the other orbital parameters remained consistent with the results of the $e_{c} \equiv 0$ model, although naturally, some parameters were subject to slightly larger uncertainties.
} 


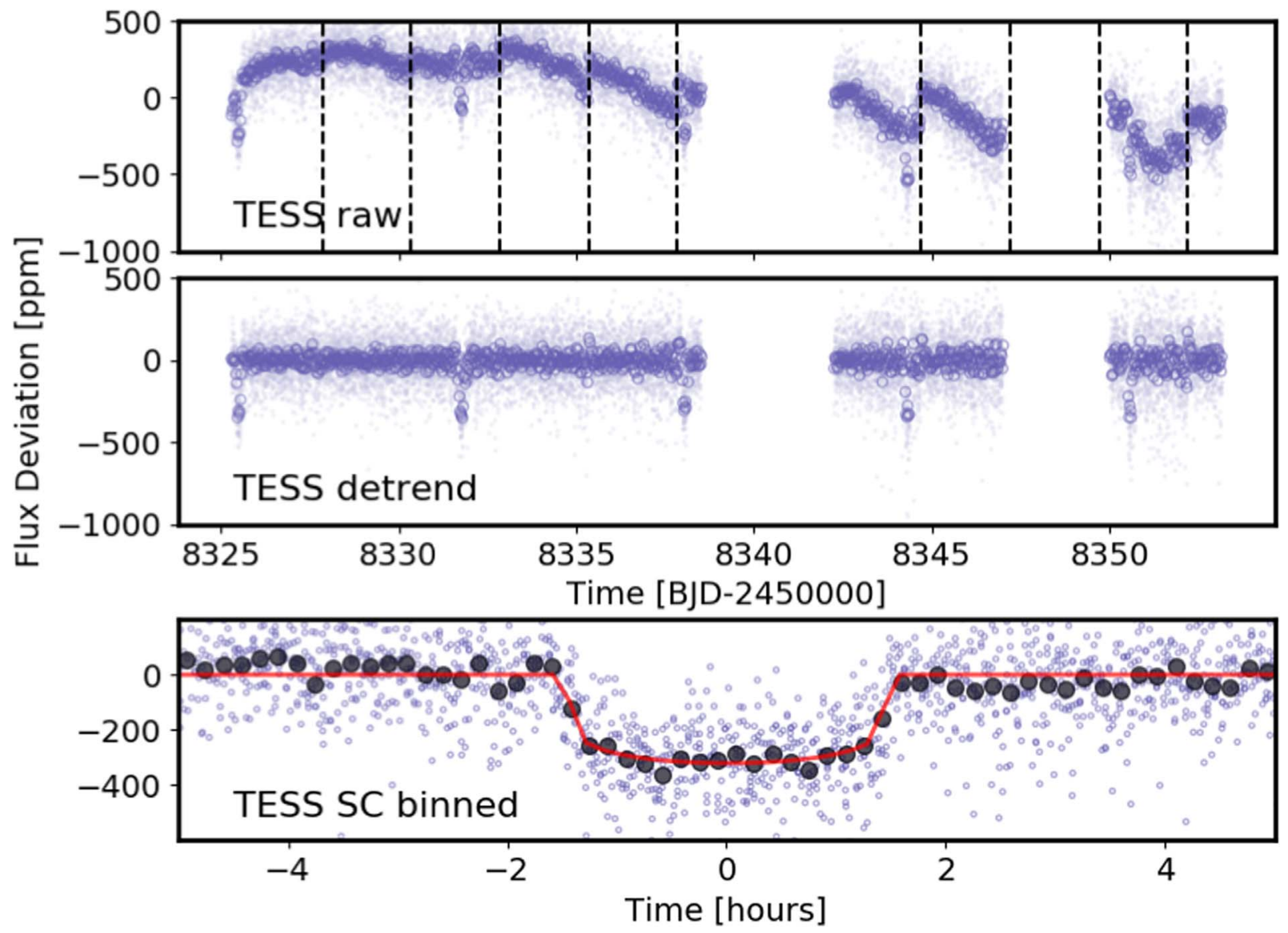

Figure 2. Raw (top panel) and corrected (middle panel) TESS light curves. The lighter points are based on the short cadence (SC) data with 2 minute sampling. The darker points are 30 minute averages. The dashed lines indicate the times of momentum dumps. The interruptions are from the data downlink and the pointing anomaly. The bottom panel shows the phase-folded light curve, along with the best-fitting model. The black dots represent 5 minute averages.

We also fitted for the mass and radius of the star, which were constrained by measurements of the spectroscopic parameters (Ghezzi et al. 2010) as well as the stellar mean density $\rho_{\star}$ implicit in the combination of $P, a / R_{\star}$, and $i$ (Seager \& MallénOrnelas 2003; Winn 2010). For a given choice of mass, age, and metallicity, we relied on the Dartmouth stellar-evolutionary models (Dotter et al. 2008) to determine the corresponding radius $R_{\star}$, effective temperature $T_{\text {eff }}$, and Gaia absolute magnitude. The likelihood function enforced agreement with the measurements of $T_{\text {eff }}, \rho_{\star}, \log g$, and parallax (based on the absolute and apparent Gaia magnitudes).

To determine the credible intervals for all of the parameters, we used the Markov Chain Monte Carlo (MCMC) method as implemented in emcee by Foreman-Mackey et al. (2013). Detrending was performed simultaneously with the transit fitting: at each step in the Markov Chain, the transit model (batman, Kreidberg 2015) was subtracted from the data and the residual light curve was detrended using a basis spline with knots spaced by 0.5 days. To avoid trying to model the discontinuities in the data related to momentum dumps, we only fitted the segment of the light curve in between momentum dumps. The results are given in Tables 1 and 2, and the best-fitting model is plotted in Figures 2 and 3. As a consistency check, we also fitted each of the five transits independently. Figure 4 shows the results, which are all consistent to within the estimated uncertainties.
Table 2

Parameters for the HD 39091 Planetary System

\begin{tabular}{|c|c|c|}
\hline Additional RV Parameters & RV offset & Instrument Jitter \\
\hline $\begin{array}{l}\text { AAT }\left(\mathrm{m} \mathrm{s}^{-1}\right) \\
\text { HARPS pre-fix }\left(\mathrm{m} \mathrm{s}^{-1}\right) \\
\text { HARPS post-fix }\left(\mathrm{m} \mathrm{s}^{-1}\right)\end{array}$ & $\begin{array}{c}32.07 \pm 0.86 \\
108.51 \pm 0.40 \\
130.60 \pm 0.70 \\
\end{array}$ & $\begin{array}{c}6.7 \pm 0.60 \\
2.33 \pm 0.18 \\
1.74 \pm 0.33 \\
\end{array}$ \\
\hline $\begin{array}{l}\text { Planet Parameters } \\
\qquad P \text { (days) } \\
T_{p}(\mathrm{BJD}) \\
T_{c}(\mathrm{BJD}) \\
K\left(\mathrm{~m} \mathrm{~s}^{-1}\right) \\
\sqrt{e} \cos \omega \\
\sqrt{e} \sin \omega \\
e \\
\omega \\
T_{14}(\mathrm{hr}) \\
a / R_{\star} \\
R_{p} / R_{\star} \\
b \equiv a \cos i / R_{\star} \\
i_{c}(\mathrm{deg})\end{array}$ & $\begin{array}{c}\text { Planet b } \\
2093.07 \pm 1.73 \\
2445852.0 \pm 3.0 \\
2446087.0 \pm 8.4 \\
192.6 \pm 1.4 \\
0.6957 \pm 0.0044 \\
-0.392 \pm 0.006 \\
0.637 \pm 0.002 \\
330.61 \pm 0.3 \\
\ldots \\
\ldots \\
\ldots \\
\ldots \\
\ldots\end{array}$ & $\begin{array}{c}\text { Planet c } \\
6.2679 \pm 0.00046 \\
\ldots \\
2458325.50400_{-0.00074}^{+0.0012} \\
1.58_{-0.28}^{+0.26} \\
\ldots \\
\ldots \\
0 \\
\ldots \\
2.953 \pm 0.047 \\
13.38 \pm 0.26 \\
0.01703_{-0.00023}^{+0.00025} \\
0.59_{-0.020}^{+0.018} \\
87.456_{-0.076}^{+0.085}\end{array}$ \\
\hline \multicolumn{3}{|l|}{ Derived Parameters } \\
\hline$M_{p} \sin i$ & $10.02 \pm 0.15 M_{\mathrm{J}}$ & $\ldots$ \\
\hline $\begin{array}{l}R_{p}\left(R_{\text {ठ }}\right) \\
\rho_{p}\left(\mathrm{~g} \mathrm{~cm}^{-3}\right) \\
\log g_{p}(\mathrm{cgs})\end{array}$ & $\begin{array}{l}\cdots \\
\cdots\end{array}$ & $\begin{array}{c}2.042 \pm 0.050 \\
2.97_{-0.55}^{+0.57} \\
3.041_{-0.86}^{+0.07}\end{array}$ \\
\hline $\begin{array}{l}a(\mathrm{AU}) \\
T_{\mathrm{eq}}(\mathrm{K})^{\mathrm{h}} \\
\left\langle F_{j}\right\rangle\left(10^{9} \mathrm{erg} \mathrm{s}^{-1} \mathrm{~cm}^{-2}\right)\end{array}$ & $\begin{array}{c}3.10 \pm 0.02 \\
\ldots \\
\ldots\end{array}$ & $\begin{array}{c}0.06839 \pm 0.00050 \\
1169.8_{-4.3}^{+2.8} \\
0.42_{-0.09}^{+0.04}\end{array}$ \\
\hline
\end{tabular}



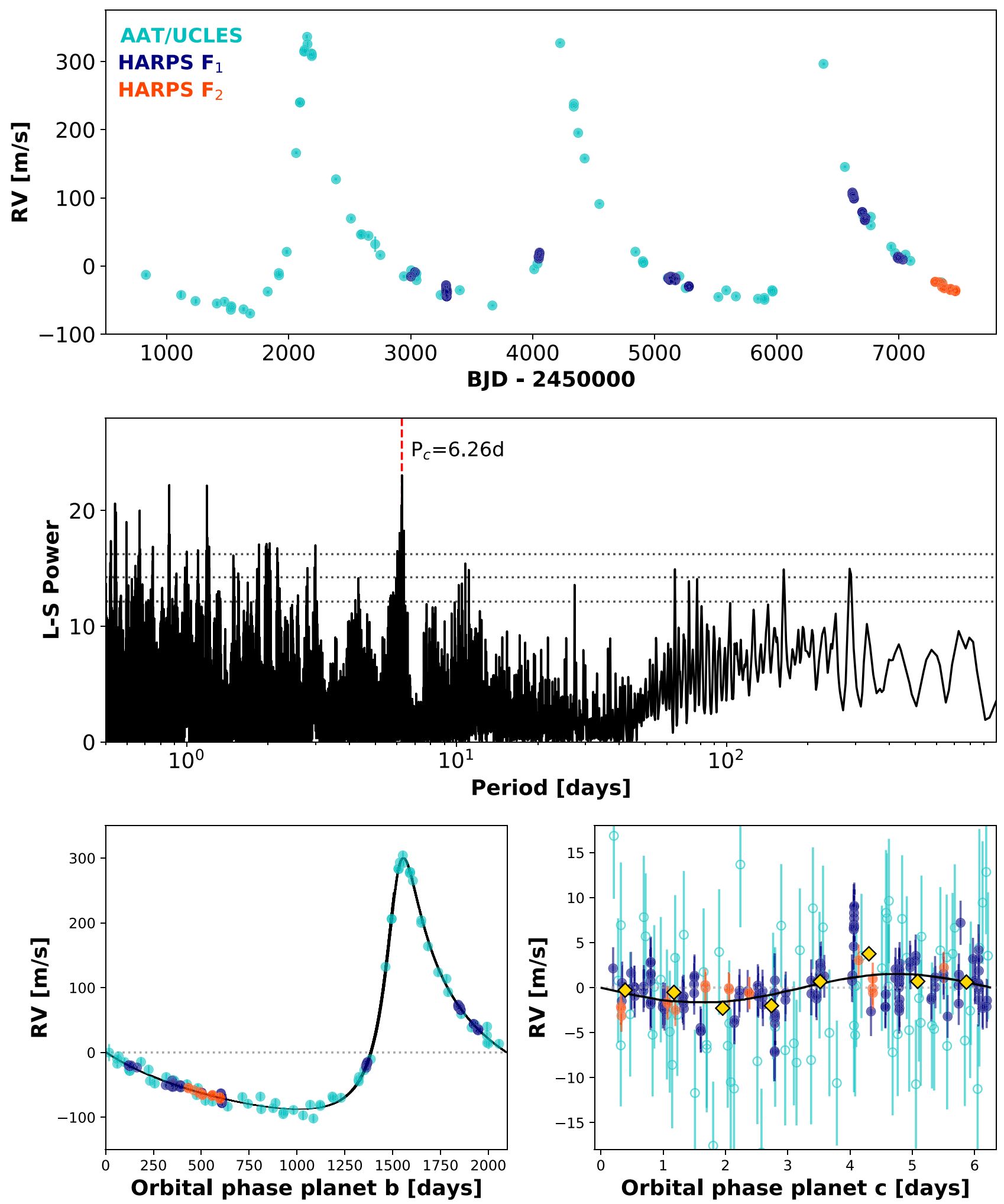

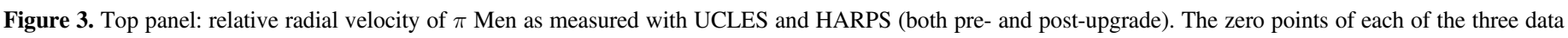

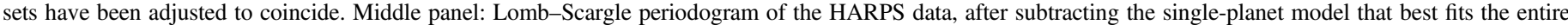

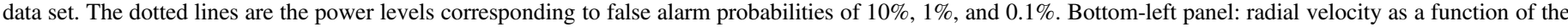

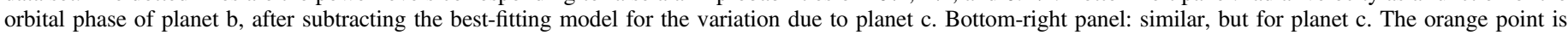
binned in phase space. 


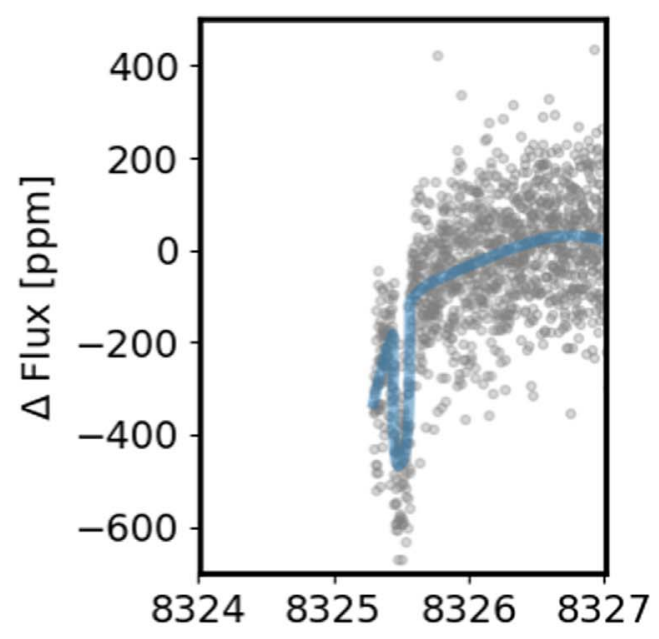

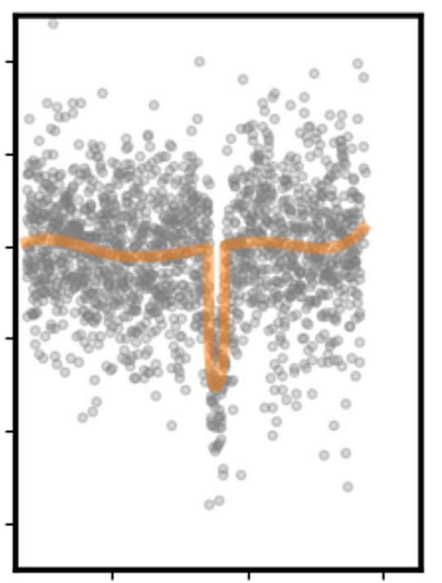

$8331 \quad 8332 \quad 8333$

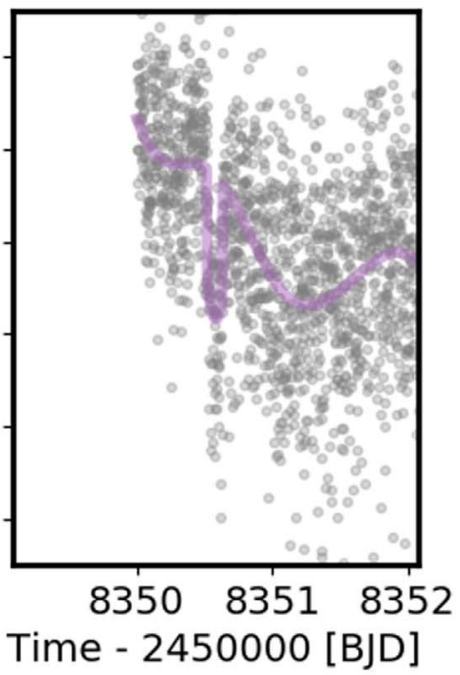

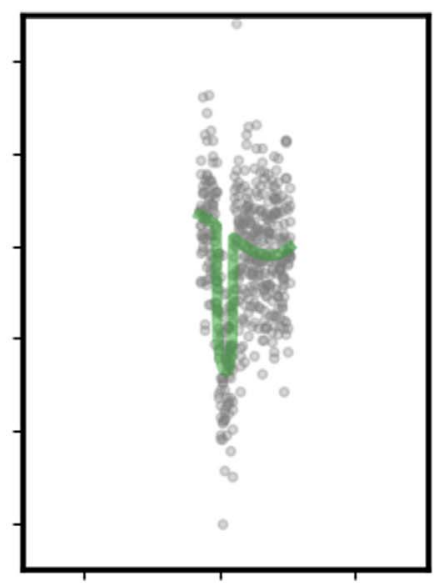
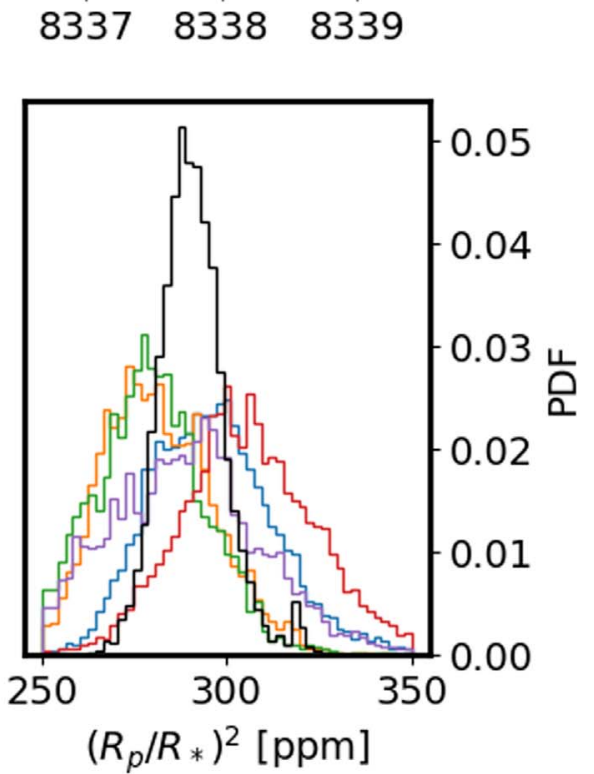

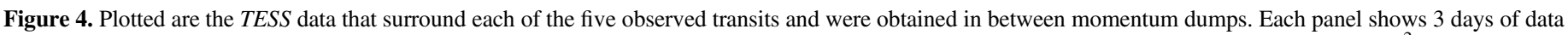

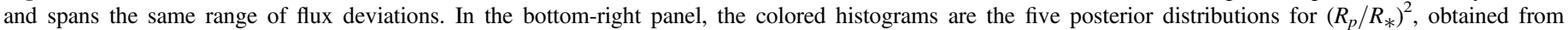
independent fits to the five transit data sets. The black histogram is the posterior based on the fit to all of the data.

\section{Discussion}

Among the known stars with transiting planets, $\pi$ Men is the second brightest in the visual band, as illustrated in the top panel of Figure 5. TESS has begun to fulfill its promise to enlarge the collection of small, transiting planets orbiting bright stars. Such stars enable precise measurements of that planet's mass and radius. The bottom panel of Figure 5 shows the measured masses and radii of the known planets smaller than Neptune, overlaid with theoretical mass/radius relationships for different compositions. $\pi$ Men c falls above the "pure rock" curve on the diagram, and near curves for planets composed of either pure water or rocky interiors surrounded by a lightweight $1 \% \mathrm{H} / \mathrm{He}$ envelope. $\pi$ Men c must not have a purely rocky composition, but instead may have a rocky core surrounded by layers of volatiles, such as hydrogen/helium (see Owen \& Wu 2017), or water/methane (Vanderburg et al. 2017).

With a near-infrared magnitude of $K=4.24, \pi$ Men is also one of the brightest stars available for planetary atmospheric characterization with the James Webb Space Telescope (JWST). $\pi$ Men $\mathrm{c}$ is one of the top 10 most favorable systems in the ranking scheme of Kempton et al. (2018), although this ranking scheme does not take into account the practical difficulties in achieving photon-limited observations of such a bright star. Transit spectroscopy would be difficult if the planet has an Earth-like atmospheric scale height of order $10 \mathrm{~km}$, in which case the atmospheric signals would be on the order of only $1 \mathrm{ppm}$. On the other hand, given the intense stellar irradiation, there may be larger signals form an escaping atmosphere (see, e.g., Ehrenreich et al. 2015; Spake et al. 2018). Spectroscopy of occultations (secondary eclipses) is also promising. The occultation depth is predicted to be $60 \mathrm{ppm}$ in the RayleighJeans limit, assuming the entire surface radiates as a blackbody at the equilibrium temperature of $1200 \mathrm{~K}$.

Another interesting possibility is to measure the stellar obliquity by observing the Rossiter-McLaughlin (RM) effect. Stars with close-in giant planets show a surprising diversity of orientations (Winn \& Fabrycky 2015; Triaud 2017). However, we know relatively little about the obliquities of stars with smaller planets, because the relevant signals are smaller and harder to detect. In the case of $\pi$ Men $\mathrm{c}$, the amplitude of the $\mathrm{RM}$ effect is on the order of $1 \mathrm{~m} \mathrm{~s}^{-1}$, the product of the transit depth $(300 \mathrm{ppm})$ and the sky-projected rotation velocity (3.1 $\mathrm{km} \mathrm{s}^{-1}$; Valenti \& Fischer 2005).

The $\pi$ Men system consists of a giant planet on a longperiod, highly eccentric orbit, along with a planet with an orbit 

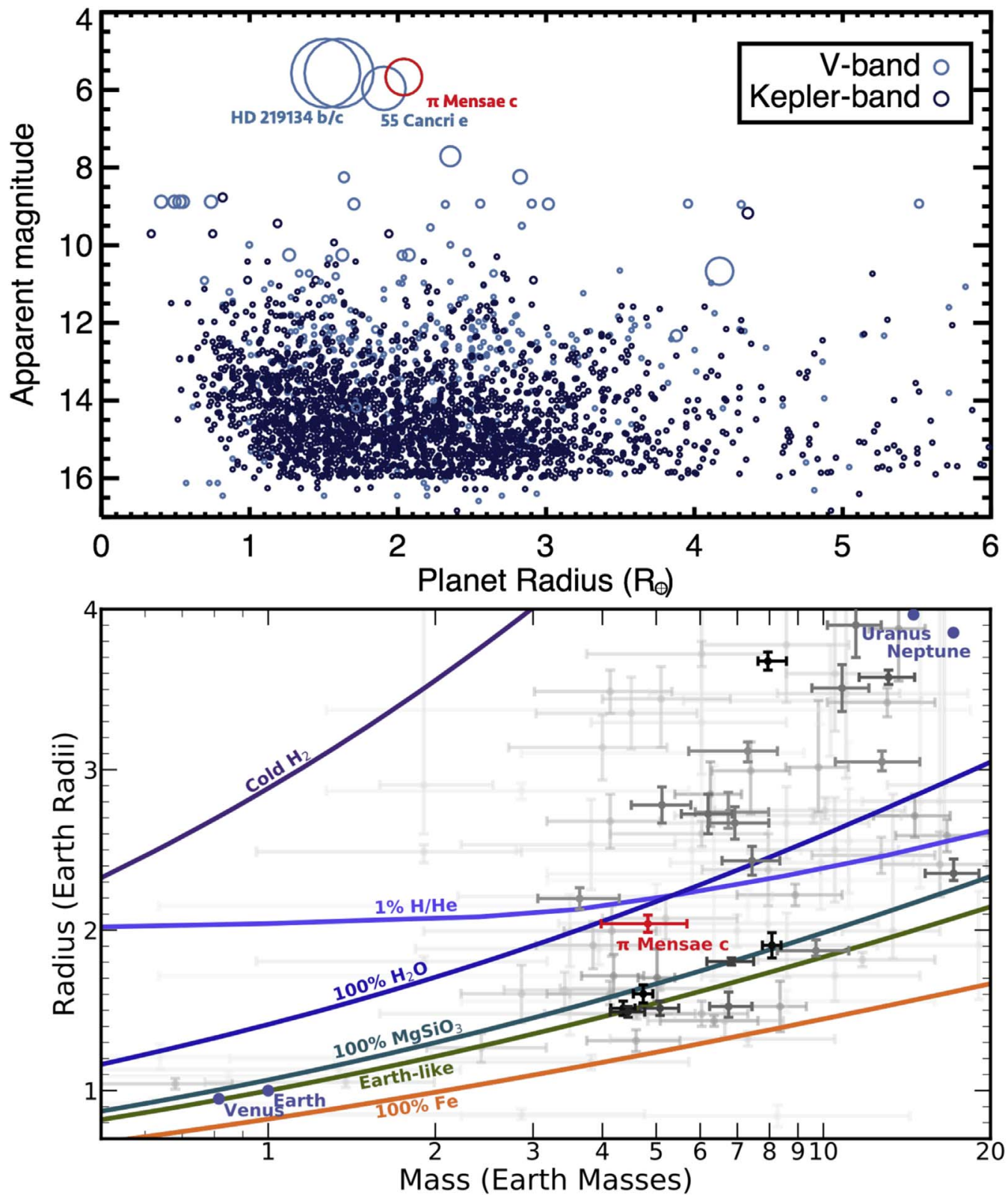

Figure 5. $\pi$ Men $\mathrm{c}$ in the context of other known exoplanets. Top panel: apparent magnitude and planet radius for all of the known transiting planets. The $V$ magnitude is plotted when available, and otherwise the Kepler magnitude is plotted. The symbol size is proportional to the angular diameter of the star. Bottom panel: massradius diagram for small exoplanets. Darker points represent more precise measurements. Based on data from the NASA Exoplanet Archive, accessed on 2018 September 13 (https://exoplanetarchive.ipac.caltech.edu/cgi-bin/TblView/nph-tblView?app=ExoTbls\&config=planets). Model curves are: $H_{2}$ (Seager et al. 2007); $100 \% \mathrm{H}_{2} \mathrm{O}, 100 \% \mathrm{M}_{g} \mathrm{~S}_{i} \mathrm{O}_{3}, 100 \% \mathrm{~F}_{e}$, Earth-like (Zeng et al. 2016); and $1 \% \mathrm{H} / \mathrm{H}_{e}$ (Lopez et al. 2012).

and mass that are both smaller by two orders of magnitude. Recent follow-up studies of Kepler systems have suggested that they maybe intrinsically common (Bryan et al. 2018; Zhu \& Wu 2018). Thus, we might find many similar cases with TESS, providing clues about the formation of close-orbiting planets, whether by disk migration, Lidov-Kozai oscillations, or other mechanisms.

Astrometric observations with the Gaia spacecraft might ultimately reveal the full 3D geometry of the system. Ranalli et al. (2018) predicted that the astrometric signal of $\pi$ Men $b$ will be detectable with a signal-to-noise ratio higher than 10 by the end of the mission. Indeed, the fit to the existing Gaia data exhibits an excess scatter of $295 \mu^{\prime \prime}(37 \sigma)$, perhaps a hint of planet-induced motion. Direct imaging might also be fruitful some day, although Zurlo et al. (2018) have already ruled out any companions with orbital separation 10-20 AU and an infrared contrast exceeding $10^{-6}$, corresponding roughly to 30 Jupiter masses.

While some of these observations may be far off, we will not have to wait long for another opportunity to learn more about $\pi$ Men. As mentioned earlier, TESS is scheduled to collect five additional months of data. This will allow us to refine our knowledge of planet $\mathrm{c}$, search for additional transiting planets, and try to detect asteroseismic oscillations. The $\pi$ Men system has already been generous to the exoplanet community, and with a little luck, the gifts will keep arriving.

An independent analysis of the TESS data has also been reported by Gandolfi et al. (2018). We acknowledge the use of TESS Alert data, which is currently in a beta test phase, from 
the TESS Science Office and at the TESS Science Processing Operations Center. Funding for the TESS mission is provided by NASA's Science Mission directorate. This research has made use of the Exoplanet Follow-up Observation Program website, which is operated by the California Institute of Technology, under contract with the National Aeronautics and Space Administration under the Exoplanet Exploration Program. C.X.H., J.B., and M.N.G. acknowledge support from MIT's Kavli Institute as Torres postdoctoral fellows. A.V.'s work was performed under contract with the California Institute of Technology/Jet Propulsion Laboratory funded by NASA through the Sagan Fellowship Program executed by the NASA Exoplanet Science Institute. J.A.D. and J.N.W. acknowledge support from the Heising-Simons Foundation. S.U., F.P., F.B., D.S., C.L., D.E., M. Marmier, and M. Mayor acknowledge financial support from the Swiss National Science Foundation (SNSF) in the frame work of the National Centre for Competence in Research Planets. D.E. acknowledges financial support from the European Research Council (ERC) under the European Unions Horizon 2020 research and innovation program (project FOUR ACES; grant agreement 724427). N.N. acknowledges partial supported by JSPS KAKENHI grant No. JP18H01265 and JST PRESTO grant No. JPMJPR1775. D.D. acknowledges support provided by NASA through Hubble Fellowship grant HSTHF2-51372.001-A awarded by the Space Telescope Science Institute, which is operated by the Association of Universities for Research in Astronomy, Inc., for NASA, under contract NAS5-26555. We made use of the Python programming language and the open-source Python packages NUMPY (van der Walt et al. 2011), EMCEE (Foreman-Mackey et al. 2013), and CELERITE (Foreman-Mackey et al. 2017). Funding for the Stellar Astrophysics Centre is provided by The Danish National Research Foundation (Grant DNRF106).

We acknowledge the traditional owners of the land on which the AAT stands, the Gamilaraay people, and pay our respects to elders past and present.

Facility: TESS, ESO:3.6m, AAT.

\section{ORCID iDs}

Chelsea X. Huang (10 https://orcid.org/0000-0003-0918-7484 Jennifer Burt (1) https://orcid.org/0000-0002-0040-6815 Andrew Vanderburg (1) https://orcid.org/0000-00017246-5438

Avi Shporer (1) https://orcid.org/0000-0002-1836-3120 Jason A. Dittmann (1) https://orcid.org/0000-0001-7730-2240 Joshua N. Winn (ib https://orcid.org/0000-0002-4265-047X Rob Wittenmyer (i) https://orcid.org/0000-0001-9957-9304 Stephen R. Kane (i) https://orcid.org/0000-0002-7084-0529 Roland K. Vanderspek (i) https://orcid.org/0000-0001-6763-6562 David W. Latham (ㄷ) https://orcid.org/0000-0001-9911-7388 Jon M. Jenkins (1) https://orcid.org/0000-0002-4715-9460 Karen A. Collins @il https://orcid.org/0000-0001-6588-9574 Samuel N. Quinn (1) https://orcid.org/0000-0002-8964-8377 David Ehrenreich (1) https://orcid.org/0000-0001-9704-5405 Edward H. Morgan (1) https://orcid.org/0000-0003-1447-6344 Jessie Christiansen (i) https://orcid.org/0000-0002-8035-4778 David Charbonneau (1) https://orcid.org/0000-00029003-484X Diana Dragomir (1) https://orcid.org/0000-0003-2313-467X
Ana Glidden (1) https://orcid.org/0000-0002-5322-2315

P. R. McCullough (1) https://orcid.org/0000-0001-9165-9799

Liang Yu (1) https://orcid.org/0000-0003-1667-5427

Norio Narita (1) https://orcid.org/0000-0001-8511-2981

Tim Morton (1) https://orcid.org/0000-0002-8537-5711

Joshua Pepper (1) https://orcid.org/0000-0002-3827-8417

András Pál (1) https://orcid.org/0000-0001-5449-2467

Joseph E. Rodriguez (10 https://orcid.org/0000-00018812-0565

Keivan G. Stassun (ib https://orcid.org/0000-0002-3481-9052 Guillermo Torres (i) https://orcid.org/0000-0002-5286-0251

Alessandro Sozzetti ib https://orcid.org/0000-0002-7504-365X Gregory Laughlin (i) https://orcid.org/0000-0002-3253-2621

G. Á. Bakos (1) https://orcid.org/0000-0001-7204-6727

Dimitar Sasselov (1) https://orcid.org/0000-0001-7014-1771

\section{References}

Bryan, M. L., Knutson, H. A., Fulton, B., et al. 2018, arXiv:1806.08799 Butler, R. P., Wright, J. T., Marcy, G. W., et al. 2006, ApJ, 646, 505 Cameron, A. C. 2012, Natur, 492, 48

Claret, A. 2017, A\&A, 600, A30

Diego, F., Charalambous, A., Fish, A. C., \& Walker, D. D. 1990, Proc. SPIE, 1235,562

Dotter, A., Chaboyer, B., Jevremović, D., et al. 2008, ApJS, 178, 89

Ehrenreich, D., Bourrier, V., Wheatley, P. J., et al. 2015, Natur, 522, 459

Foreman-Mackey, D., Agol, E., Ambikasaran, S., \& Angus, R. 2017, AJ, 154,220

Foreman-Mackey, D., Hogg, D. W., Lang, D., \& Goodman, J. 2013, PASP, 125,306

Gaia Collaboration, Brown, A. G. A., Vallenari, A., et al. 2018, A\&A, 616, A1 Gandolfi, D., Barragan, O., Livingston, J., et al. 2018, arXiv:1809.07573 Ghezzi, L., Cunha, K., Smith, V. V., et al. 2010, ApJ, 720, 1290

Günther, M. N., Queloz, D., Gillen, E., et al. 2017, MNRAS, 472, 295

Günther, M. N., Queloz, D., Gillen, E., et al. 2018, MNRAS, 478, 4720

Hadden, S., \& Lithwick, Y. 2017, AJ, 154, 5

Jenkins, J. M., Caldwell, D. A., Chandrasekaran, H., et al. 2010, ApJL, 713, L87

Jones, H. R. A., Paul Butler, R., Tinney, C. G., et al. 2002, MNRAS, 333, 871 Kempton, E. M.-R., Bean, J. L., Louie, D. R., et al. 2018, PASP, 130, 114401 Kreidberg, L. 2015, PASP, 127, 1161

Lo Curto, G., Pepe, F., Avila, G., et al. 2015, Msngr, 162, 9

Lopez, E. D., Fortney, J. J., \& Miller, N. 2012, ApJ, 761, 59

Mandel, K., \& Agol, E. 2002, ApJL, 580, L171

Mayor, M., Pepe, F., Queloz, D., et al. 2003, Msngr, 114, 20

Owen, J. E., \& Wu, Y. 2017, ApJ, 847, 29

Pál, A. 2009, PhD thesis, Eötvös Loránd Univ.

Ranalli, P., Hobbs, D., \& Lindegren, L. 2018, A\&A, 614, A30

Ricker, G. R., Winn, J. N., Vanderspek, R., et al. 2015, JATIS, 1, 014003

Seager, S., Kuchner, M., Hier-Majumder, C. A., \& Militzer, B. 2007, ApJ, 669,1279

Seager, S., \& Mallén-Ornelas, G. 2003, ApJ, 585, 1038

Spake, J. J., Sing, D. K., Evans, T. M., et al. 2018, Natur, 557, 68

Stassun, K. G., Oelkers, R. J., Pepper, J., et al. 2018, AJ, 156, 102

Triaud, A. H. M. J. 2017, in Handbook of Exoplanets, ed. H. J. Deeg \& J. A. Belmonte (Dordrecht: Springer), 2

Valenti, J. A., \& Fischer, D. A. 2005, ApJS, 159, 141

van der Walt, S., Colbert, S. C., \& Varoquaux, G. 2011, CSE, 13, 22

Van Eylen, V., \& Albrecht, S. 2015, ApJ, 808, 126

Vanderburg, A., Becker, J. C., Buchhave, L. A., et al. 2017, AJ, 154, 237

Winn, J. N. 2010, in Exoplanet Transits and Occultations, ed. S. Seager (Univ. Arizona Press), 55

Winn, J. N., \& Fabrycky, D. C. 2015, ARA\&A, 53, 409

Wittenmyer, R. A., Horner, J., Tuomi, M., et al. 2012, ApJ, 753, 169

Zeng, L., Sasselov, D. D., \& Jacobsen, S. B. 2016, ApJ, 819, 127

Zhu, W., \& Wu, Y. 2018, AJ, 156, 92

Zurlo, A., Mesa, D., Desidera, S., et al. 2018, MNRAS, 480, 35 